\title{
Avaliabilidade da Politica Nacional de Regulação no SUS: uma proposta preliminar
}

\author{
| ${ }^{1}$ Tatiana Aragão Figueiredo, ${ }^{2}$ Antonia Angulo-Tuesta, ${ }^{3}$ Zulmira Hartz |
}

Resumo: Os estudos de avaliabilidade permitem determinar os propósitos e o foco avaliativo de políticas, auxiliando o entendimento aprofundado e a apreciação prévia das possibilidades de avaliação. Assim, este artigo busca definir em que medida a Política Nacional de Regulação está em condiçôes de ser avaliada a partir da descrição, elaboraçáo dos modelos lógico e teórico de avaliação, de acordo com o sistema dos sete elementos de Thurston e Ramaliu. Trata-se de estudo de abordagem exploratória e qualitativa, desenvolvido de janeiro a maio de 2018. Os resultados contribuíram para a melhor compreensão da estrutura e operacionalização desta política. Essa Política, entretanto, conforme apresentada e discutida, possui numerosos componentes e atividades, sendo difícil a elaboraçáo de indicadores e de matriz de avaliaçáo. Sugere-se um recorte da política por dimensão ou em menor escala para o possível processo avaliativo. Cabe pontuar que após dez anos de existência, a Política Nacional de Regulação não recebeu proposiçôes de avaliação. Sabe-se que a regulação no setor saúde apresenta diversos desafios, sendo necessária uma proposta para além das dimensôes de eficiência e equidade, considerando a formação e as atividades dos profissionais, a acessibilidade aos serviços e as tecnologias em saúde.

> Palavras-chave: avaliação em saúde; políticas de saúde; regulação e fiscalização em saúde; sistema de saúde.

\author{
1 Instituto de Tecnologia em \\ Fármacos, Núcleo de Inovação \\ Tecnológica. Rio de Janeiro-RJ, \\ Brasil (tatiana.figueiredo@far. \\ fiocruz.br) \\ ORCID: 0000-0002-3932-0453 \\ 2 Programa de Pós-Graduação \\ em Ciências e Tecnologias \\ em Saúde, Universidade de \\ Brasilia. Ceilândia-GO, Brasil \\ (antoniaangulo@unb.br). \\ ORCID: 0000-0002-3231-5918. \\ ${ }^{3}$ Instituto de Higiene e Medicina \\ Tropical, Universidade Nova de \\ Lisboa. Lisboa, Portugal (zhartz@ \\ inmt.unl.pt). \\ ORCID: 0000-0001-9780-9428.
}

Recebido em: 20/12/2018 Revisado em: 21/02/2019 Aprovado em: 27/02/2019 


\section{Introdução}

A regulação inclui tanto o ato de regulamentar e elaborar leis, regras ou normas, quanto as açóes e técnicas que asseguram o cumprimento dessas leis, quais sejam, fiscalização, controle, avaliação, auditoria, sanções e premiações (SCHILLING et al., 2006). Uma vez que o Estado pode transferir açôes para o setor privado ou agir em parceria com agentes sociais, deixa de ser o centralizador da produção do bem público e passa a ser o coprodutor, mantendo a condição de responsável último por sua produção. Neste raciocínio, a regulação pode ser vista como a influência do Estado em qualquer área ou setor da sociedade. Pode-se dizer que o termo "regulação" refere-se aos serviços que funcionam sob concessão do Estado para suprir necessidades da população, e o papel do Estado, ao se utilizar da regulação, define os critérios de organização e prestação dos serviços, estabelecendo as prioridades e regras para atuação dos mercados (VILARINS et al., 2012).

Segundo Salgado (2003), a regulação pode ser entendida como instrumento essencial para a manutenção do equilíbrio de qualquer sistema. Sendo atribuição dos órgãos reguladores, permite identificar as perturbaçóes geradas, analisar e tratar as informaçôes, e transmitir um conjunto de ordens coerentes a um ou a vários dos seus órgãos executores (BARROSO, 2005). Tem-se assim um sistema que funciona a partir de regras e parâmetros acordados entre as partes executoras e reguladoras (VILARINS et al., 2012).

No setor saúde, a regulação compreende açôes de regulamentação, fiscalização, controle, auditoria e avaliaçáo de determinado sujeito social sobre a produção e a distribuição de bens e serviços de saúde. Tem como objeto os estabelecimentos, envolvendo estrutura física, equipamentos, profissionais, entre outros; as relaçóes contratuais; o exercício das profissões de saúde; a oferta e a demanda por serviços; os protocolos assistenciais; os fluxos de atendimento; a produção, a venda, a incorporação e o uso de insumos, medicamentos e de outras tecnologias; as condições de trabalho e os ambientes relativos ao setor saúde; além do controle e da avaliação dos custos e gastos em saúde. Assim, pode-se dizer que a regulação no setor saúde é uma ação política de garantia de direitos sobre os interesses do mercado (BRASIL, 2016a; GIANNOTTI, 2013; SCHILLING et al., 2006).

No cenário brasileiro, o Ministério da Saúde (MS) instituiu a Política Nacional de Regulação (PNR) em 2008 (BRASIL, 2008), introduzindo o conceito ampliado 
de regulação a partir de três eixos: segundo a sua atuação nos sistemas de saúde (regulação sobre sistemas), sobre a produção direta das açóes e serviços de saúde (regulação da atenção) e sobre o acesso dos usuários aos serviços de saúde (regulação do acesso). Esse conceito associa e articula as açôes de regulação em saúde ao monitoramento, controle, avaliação, auditoria e vigilância da atenção e da assistência à saúde no âmbito do SUS (ALBIERI; CECILIO, 2015).

Essa política resultou de iniciativas, a partir da década de 1990, que definiram os papéis dos níveis de gestão no processo regulatório das normas operacionais: a regulamentação dos planos privados de saúde (Lei no 9.656/1998) e as criaçôes da Agência Nacional de Vigilância Sanitária - Anvisa (Lei no 9.782/1999) e da Agência Nacional de Saúde Suplementar - ANS (Lei no 9.961/2000).

A partir de 2001, em um contexto de regionalização, a regulação da assistência aprofunda-se, introduzindo o conceito de "regulação assistencial" ou de "regulação do acesso às urgências". Em 2006, ganha força com o Pacto pela Saúde, que instituiu diretrizes para a consolidação de processos de regionalização, a partir de instrumentos de planejamento e programação, que orientavam a descentralização, e da reorganização da gestão e regulação do sistema de saúde (CONASS, 2011; MACHADO, 2007).

Segundo Leviton et al. (2010), a implantação de uma política ou programa evidencia o quanto essa intervençấo se encontra adequadamente operacionalizada, e a avaliação dessa implantação permite conhecer a totalidade das intervençôes no que tange à validade do seu conteúdo e aos fatores que explicam possíveis defasagens entre a planificação e a execução das açôes. Para tal, faz-se necessário construir a teoria do programa, o contexto e resultados esperados (HARTZ; VIEIRA-DASILVA, 2005). Todavia, antes de iniciar a avaliação de uma política ou de um programa, sugere-se realizar um estudo exploratório da situação concreta, isto é, a prévia da avaliação, como é o caso do estudo de avaliabilidade. Em termos teóricos e práticos, utiliza-se tal estudo para determinar se há justificativa para uma avaliação, com vistas a delimitar os objetivos da política, bem como desvelar áreas críticas que demandam foco e atenção (SILVA, 2014).

Alguns estudos avaliativos foram realizados a fim de avaliar o acesso a leitos (ALBUQUERQUE, 2010), a implantação de complexos reguladores (FERREIRA et al., 2010), a implantação de central de regulação médica (DIAS, 2012) e a qualidade regulatória das agências reguladoras (SILVA, 2013; ALVES, PECI 2011). 
$\mathrm{Na}$ verdade, discute-se muito como avaliar a regulação nas dimensôes acesso, qualidade dos serviços e concorrência dos segmentos abertos ao mercado (SIMÓES, 2017). Também foram identificados estudos teóricos acerca dos conceitos de regulação e da Política de Regulação (VILARINS et al., 2012; BARBOSA et al., 2016; OLIVEIRA; ELIAS, 2012; PEITER et al., 2016), mas não estudos que se propusessem a avaliar a referida política.

Neste contexto e considerando-se que no sistema de saúde brasileiro (Sistema Único de Saúde - SUS) disputam e convivem duas lógicas distintas: a pública e de acesso universal, financiada por impostos e contribuiçóes sociais pagos por toda a sociedade; e a privada, relativa ao sistema de saúde suplementar, de acesso restrito a parte da população, custeada pelas mensalidades pagas pelos clientes individuais e principalmente por empresas que financiam planos de saúde coletivos para seus funcionários. Sendo assim, torna-se relevante realizar um estudo de avaliabilidade da PNR com o objetivo de definir em que medida esta política tem condiçôes de ser foco de avaliação a partir da descrição da mesma e da elaboração dos modelos lógico e teórico de avaliação (GUETTERMAN; HARE, 2014; NASCIMENTO et al., 2014; BRASIL, 2016a).

\section{Metodologia}

Realizou-se estudo de avaliabilidade, no período de janeiro a maio de 2018, de abordagem exploratória e qualitativa, de acordo com o sistema dos sete elementos de Thurston e Ramaliu (2005), a saber: (1) delimitação da política e identificação de suas metas, objetivos e atividades; (2) identificação e análise dos documentos da intervenção a ser avaliada; (3) construção do modelo lógico; (4) supervisão da política ou entendimento preliminar de como a política opera; (5) desenvolvimento de um modelo teórico de avaliação; (6) identificação dos usuários e envolvidos na avaliação; e (7) definição de procedimento de avaliação.

Neste estudo não foi desenvolvido o sétimo elemento, inviabilizando a formulação de perguntas avaliativas não foram formuladas, devido à limitação do tempo para a definição de usuários, análise e validação dos modelos lógico e teórico do programa.

A operacionalização do estudo baseou-se em pesquisa bibliográfica e análise documental da PNR do SUS, das políticas relacionadas, decretos de criação e redefinição, e das portarias que a normatizam. 
As bases de dados eletrônicas utilizadas foram: Saúde Legis (http://portal2. saude.gov.br/saudelegis/LEG_NORMA_PESQ_CONSULTA.CFM), portal do MS (http://portalms.saude.gov.br/), MEDLINE (Medical Literature Analysis and Retrieval System Online) e LILACS (Literatura Latino-Americana e do Caribe em Ciências da Saúde). Além disso, foi realizada busca manual de publicaçôes com base nas referências bibliográficas identificadas.

A estratégia de busca utilizou como descritores as seguintes expressóes: "regulação e fiscalização em saúde”; "regulação em saúde”; "Política Nacional de Regulação do Sistema Único de Saúde"; "central de regulação" e "complexos reguladores”. Foram selecionados os trabalhos publicados entre 2008 a 2017, período compreendido entre a publicação dessa política e a execução deste estudo. $\mathrm{O}$ quadro 1 apresenta o resumo das fontes de dados básicas para a realizaçáo do estudo de avaliabilidade e as contribuiçôes em cada elemento de Thurston e Ramaliu (2005).

Quadro 1. Resumo das fontes de dados e as contribuiçōes em cada elemento, segundo Thurston e Ramaliu (2005). Rio de Janeiro, 2018

\begin{tabular}{|l|l|l|}
\hline Elementos & Contribuiçóes & Fonte de dados \\
\hline $\begin{array}{l}\text { 1. Delimitação } \\
\text { da política e } \\
\text { identificação de suas } \\
\text { metas, objetivos e } \\
\text { atividades. }\end{array}$ & $\begin{array}{l}\text { Análise das normas legais e } \\
\text { diretrizes, a fim de verificar } \\
\text { a existência de objetivos, } \\
\text { metas, atividades das três } \\
\text { dimensóes da PNR. }\end{array}$ & $\begin{array}{l}\text { Portaria GM/MS no 1.559, de } \\
\text { agosto de 2008 (BRASIL, 2008). }\end{array}$ \\
\hline $\begin{array}{l}\text { 2. Identificação } \\
\text { e análise dos } \\
\text { documentos da } \\
\text { intervenção a ser } \\
\text { avaliada. }\end{array}$ & $\begin{array}{l}\text { Análise da adequação, } \\
\text { contexto, conceitos-chave, } \\
\text { factibilidade, coerência e } \\
\text { viabilidade dos documentos } \\
\text { normativos e oficiais ligados } \\
\text { à PNR. }\end{array}$ & $\begin{array}{l}\text { • Portaria GM/MS no 1.559, de } \\
\text { agosto de 2008 (BRASIL, 2008). } \\
\text { Sistema Único de Saúde - SUS } \\
\text { (BRASIL, 2016a). }\end{array}$ \\
\hline
\end{tabular}

continua... 


\begin{tabular}{|c|c|c|}
\hline Elementos & Contribuiçôes & Fonte de dados \\
\hline $\begin{array}{l}\text { 3. Construção do } \\
\text { modelo lógico. }\end{array}$ & $\begin{array}{l}\text { Identificação das relações } \\
\text { entre os insumos, as } \\
\text { atividades vinculadas às } \\
\text { dimensões da política e os } \\
\text { resultados esperados. }\end{array}$ & $\begin{array}{l}\text { - Portaria GM/MS no 1.559, de } \\
\text { agosto de 2008 (BRASIL, 2008). } \\
\text { - Curso Básico de Regulação do } \\
\text { Sistema Único de Saúde - SUS } \\
\text { (BRASIL, 2016a). } \\
\text { - Diretrizes para a Implantação de } \\
\text { Complexos Reguladores (BRASIL, } \\
\text { 2006). } \\
\text { - Manual de orientaçóes para } \\
\text { Contrataçáo de Serviços de Saúde } \\
\text { (BRASIL, 2016b). } \\
\text { - Orientaçóes Básicas para utilização } \\
\text { de Sistemas informatizados em } \\
\text { auditoria no SUS (BRASIL, 2007). } \\
\text { - Sistema de Informação Hospitalar } \\
\text { do SUS 2014-SIH: Manual } \\
\text { Técnico Operacional do Sistema. } \\
\text { Brasília (BRASIL, 2015). } \\
\text { - Manual das ouvidorias do SUS } \\
\text { (BRASIL, 2014). }\end{array}$ \\
\hline $\begin{array}{l}\text { 4. Supervisão } \\
\text { da política, ou } \\
\text { entendimento } \\
\text { preliminar de como a } \\
\text { política opera. }\end{array}$ & $\begin{array}{l}\text { Explicitação da organização } \\
\text { e do funcionamento das } \\
\text { dimensóes da PNR, a fim } \\
\text { de identificar as atividades } \\
\text { necessárias para a obtenção } \\
\text { dos resultados. }\end{array}$ & $\begin{array}{l}\text { - Portaria GM/MS no } 1.559 \text {, de } \\
\text { agosto de } 2008 \text { (BRASIL, 2008). }\end{array}$ \\
\hline $\begin{array}{l}\text { 5. Desenvolvimento } \\
\text { de um modelo } \\
\text { teórico de avaliação. }\end{array}$ & $\begin{array}{l}\text { Descrição da teoria da } \\
\text { política e identificação das } \\
\text { relaçóes entre o contexto } \\
\text { político-institucional, os } \\
\text { componentes do modelo } \\
\text { lógico e os cenários da } \\
\text { implantação da política. }\end{array}$ & $\begin{array}{l}\text { - Portaria GM/MS no 1.559, de } \\
\text { agosto de } 2008 \text { (BRASIL, 2008). } \\
\text { - Curso Básico de Regulação do } \\
\text { Sistema Único de Saúde - SUS } \\
\text { (BRASIL, 2016a). }\end{array}$ \\
\hline $\begin{array}{l}\text { 6. Identificação dos } \\
\text { usuários a serem } \\
\text { envolvidos na } \\
\text { avaliação. }\end{array}$ & $\begin{array}{l}\text { Identificação do público-alvo; } \\
\text { pactuaçáo do plano de } \\
\text { avaliaçáo. }\end{array}$ & $\begin{array}{l}\text { - Portaria GM/MS no } 1.559 \text {, de } \\
\text { agosto de } 2008 \text { (BRASIL, 2008). }\end{array}$ \\
\hline
\end{tabular}




\section{Resultados}

\section{Compreendendo a Intervenção (elementos 1, 2 e 4)}

A atuação do mercado no setor Saúde levanta muitos argumentos contrários, podendo-se destacar sua incapacidade de produzir equidade. Isso porque o mercado náo requer padrão de distribuição de renda; ao contrário, reforça o padrão existente. $\mathrm{O}$ mercado exacerba as inequidades, pela alocação de bens e serviços de acordo com a capacidade individual paga por eles (BRASIL, 2016a; OCKÉ-REIS, 2018).

Entre os desafios da regulação em saúde, destacam-se a compreensão da multiplicidade de cenários, sujeitos, ações e interesses, e a implementação de estratégias de regulação centradas no usuário, a fim de lhe garantir os direitos constitucionais tratados no capítulo da saúde (BRASIL, 2016a; SCHILLING et al., 2006). Neste sentido, diversos fatores justificam a necessidade de regulação estatal no setor Saúde: 1) corrigir/atenuar as falhas de mercado na produção e distribuição de bens e serviços de saúde; 2) resolver o problema do uso inadequado ou da introdução de novas tecnologias sem critérios no sistema de saúde; 3) planejar a oferta de bens e serviços de saúde, de acordo com as necessidades da população, e não em função de interesses individuais ou pressóes de determinados grupos; 4) garantir padróes de qualidade dos serviços prestados à população; e 5) organizar um modelo de atenção à saúde que seja mais eficiente e resolutivo.

No Brasil, historicamente, as ações de controle e avaliação no SUS deram-se sobre: 1) a execução das ações diretas de saúde como consultas, exames, entre outros, com foco na contabilidade financeira do pagamento da produção e/ou nos processos de execução das açôes, ou seja, envolvendo os prestadores de serviços e, assim, seu cadastro, habilitação, autorização etc.; 2) a execução orçamentária e a aplicação dos recursos destinados à saúde, segundo cada esfera de gestão; 3) a produção e a comercialização de serviços, produtos e substâncias de interesse para a saúde; e 4) o desempenho dos sistemas de saúde em cada esfera de gestão do SUS (BRASIL, 2016a; SCHILLING et al., 2006; GIANNOTTI, 2013).

No entanto, a articulação entre essas ações nem sempre ocorreu, havendo certa separação, ou seja, especialização de cada ação e seu respectivo agente. $\mathrm{O}$ controle e a avaliação tiveram como ação principal o controle da execução das ações diretas de saúde; a auditoria priorizou a execução orçamentária, a aplicação dos recursos 
destinados à saúde, além de verificar a regularidade dos serviços mediante o exame analítico e pericial; a vigilância sanitária fiscalizou a produção e a comercialização de serviços, produtos e substâncias de interesse para a saúde; e à ANS coube regular o sistema de saúde suplementar. Ao mesmo tempo, existiu certa confusão e sobreposição das competências, porque quase todos fizeram do sistema seu objeto de ação. Por exemplo, a auditoria também passou a analisar a execução das ações diretas de saúde, ação esta que era do controle e avaliação (SCHILLING et al., 2006; GIANNOTTI, 2013).

Em meio a tal sobreposição e ao desenvolvimento de políticas de regulação que já vinham se estruturando no Brasil, foi publicada a Portaria GM/MS no 1.559, de agosto de 2008, instituindo a PNR (BRASIL, 2008). Seus objetivos são estruturar as ações de regulação, controle e avaliação no SUS, fortalecer a regionalização, hierarquização e integração das açóes e serviços, integrar e aprimorar os processos de trabalho e fortalecer os mecanismos e estratégias de gestão. As açóes foram organizadas em três dimensôes, que devem funcionar de maneira integrada: a Regulação de Sistemas de Saúde, a Regulação da Atenção à Saúde e a Regulação do Acesso à Assistência.

A Regulação de Sistemas de Saúde tem como objeto os sistemas municipal, estadual e nacional de saúde, e como objetivo a definição de macrodiretrizes para regulação da atenção, a partir dos princípios e diretrizes do SUS. Suas açôes são: 1) elaboração de decretos, normas e portarias que dizem respeito às funçôes de gestão; 2) planejamento, financiamento e fiscalização de sistemas de saúde; 3) controle social e ouvidoria em saúde; 4) vigilância sanitária e epidemiológica; 5) regulação da saúde suplementar; 6) auditoria assistencial ou clínica; e 7) avaliação e incorporação de tecnologias em saúde (BRASIL, 2008).

A Regulação da Atenção à Saúde, exercida pelas secretarias estaduais e municipais de Saúde, tem como objeto a produção das ações diretas e finais de atenção à saúde, e como objetivo a garantia da adequada prestação de serviços à população. Suas açóes englobam: 1) cadastramento de estabelecimentos e profissionais de saúde no Sistema de Cadastro Nacional de Estabelecimentos de Saúde (SCNES); 2) cadastramento de usuários do SUS no sistema do Cartão Nacional de Saúde (CNS); 3) contratualização de serviços de saúde segundo as normas e políticas específicas do MS; 4) credenciamento/habilitação para a prestação de serviços de saúde; 5) elaboração e 
incorporação de protocolos de regulação que ordenam os fluxos assistenciais; 6) supervisão e processamento da produção ambulatorial e hospitalar; 7) atividades da Programação Geral das Ações e Serviços de Saúde (PGASS); 8) avaliação analítica da produção; 9) avaliação de desempenho dos serviços e da gestão e de satisfação dos usuários (Pnass); 10) avaliação das condiçôes sanitárias dos estabelecimentos de saúde; 11) avaliação dos indicadores epidemiológicos e das ações e serviços de saúde nos estabelecimentos de saúde; e 12) utilização de sistemas de informação que subsidiam os cadastros, a produção e a regulação do acesso (BRASIL, 2008).

Por fim, a Regulação do Acesso à Assistência, também denominada regulação do acesso ou regulação assistencial, tem como objetos a organização, o controle, o gerenciamento e a priorização do acesso e dos fluxos assistenciais no âmbito do SUS, e como objetivo efetivar a disponibilização da alternativa assistencial mais adequada à necessidade do cidadão, por meio de atendimentos às urgências, consultas, leitos, apoio diagnóstico e terapias (portaria). Sendo assim, esta dimensão abrange a regulação médica, exercendo autoridade sanitária para a garantia do acesso baseada em protocolos, classificação de risco e demais critérios de priorização. Suas ações envolvem: 1) regulação do acesso a partir da atenção básica, unidades especializadas e atenção pré-hospitalar e hospitalar às urgências; 2) controle dos leitos disponíveis e das agendas de consultas e procedimentos especializados; 3) autorização dos procedimentos de alto custo/complexidade; 4) padronização das solicitaçôes de procedimentos por meio dos protocolos de regulação do acesso; 5) estabelecimento de referências entre unidades de diferentes níveis de complexidade, de abrangência local, intermunicipal e interestadual, segundo fluxos e protocolos pactuados (BRASIL, 2008).

Segundo a PNR (BRASIL, 2008), a regulação do acesso organiza-se por meio de complexos reguladores e centrais de regulação. A Central de Regulação Ambulatorial regula o acesso a todos os procedimentos ambulatoriais, incluindo terapias e cirurgias ambulatoriais. A Central de Regulação Hospitalar regula o acesso aos leitos e aos procedimentos hospitalares eletivos e, conforme organização local, o acesso aos leitos hospitalares de urgência. A Central de Regulação de Urgências regula o atendimento pré-hospitalar de urgência e, conforme organização local, o acesso aos leitos hospitalares de urgência. A Central Estadual de Regulação da Alta Complexidade foi integrada às Centrais de Regulação de 
Consultas e Exames e Internações Hospitalares e à Central Nacional de Regulação de Alta Complexidade/Custo.

Importante destacar que nem a PNR, nem os demais documentos analisados apresentam os objetivos, as metas e as estratégias de supervisão da política, constando apenas suas atividades.

\section{Modelo Lógico da Política Nacional de Regulação (elemento 3)}

Tendo percorrido as etapas de exploraçáo dos documentos relativos à institucionalização e implantação da PNR, prosseguiu-se à análise, orientada pelo referencial de avaliabilidade com a apresentação do modelo lógico (THURSTON; RAMALIU, 2005).

O modelo lógico retrata um quadro que pode ser uma referência para ajudar todos os envolvidos na implementação da PNR. Desta forma, cria-se a possibilidade de rever a política, reforçando a necessidade de um processo participativo, ouvindo gestores e executores das açóes nos territórios (DIAS et al., 2018).

Uma análise profunda é dificultada pela falta de especificaçôes dos documentos oficiais. Silva (2007), a partir da análise da teoria de programa, destaca a importância do modelo lógico para a identificação de deficiências ou problemas em seu fluxo, que podem interferir no seu desempenho. Avaliar a qualidade da teoria significa verificar se o programa está bem desenhado e se apresenta um plano plausível de alcance dos resultados esperados.

O objetivo de um modelo lógico é ser um desenho funcional que represente um programa/política e sua possível execução, além de resolver os problemas iniciais identificados em um determinado cenários (DIAS et al., 2018). A figura 1 apresenta o modelo lógico da PNR, de acordo com suas dimensôes. 


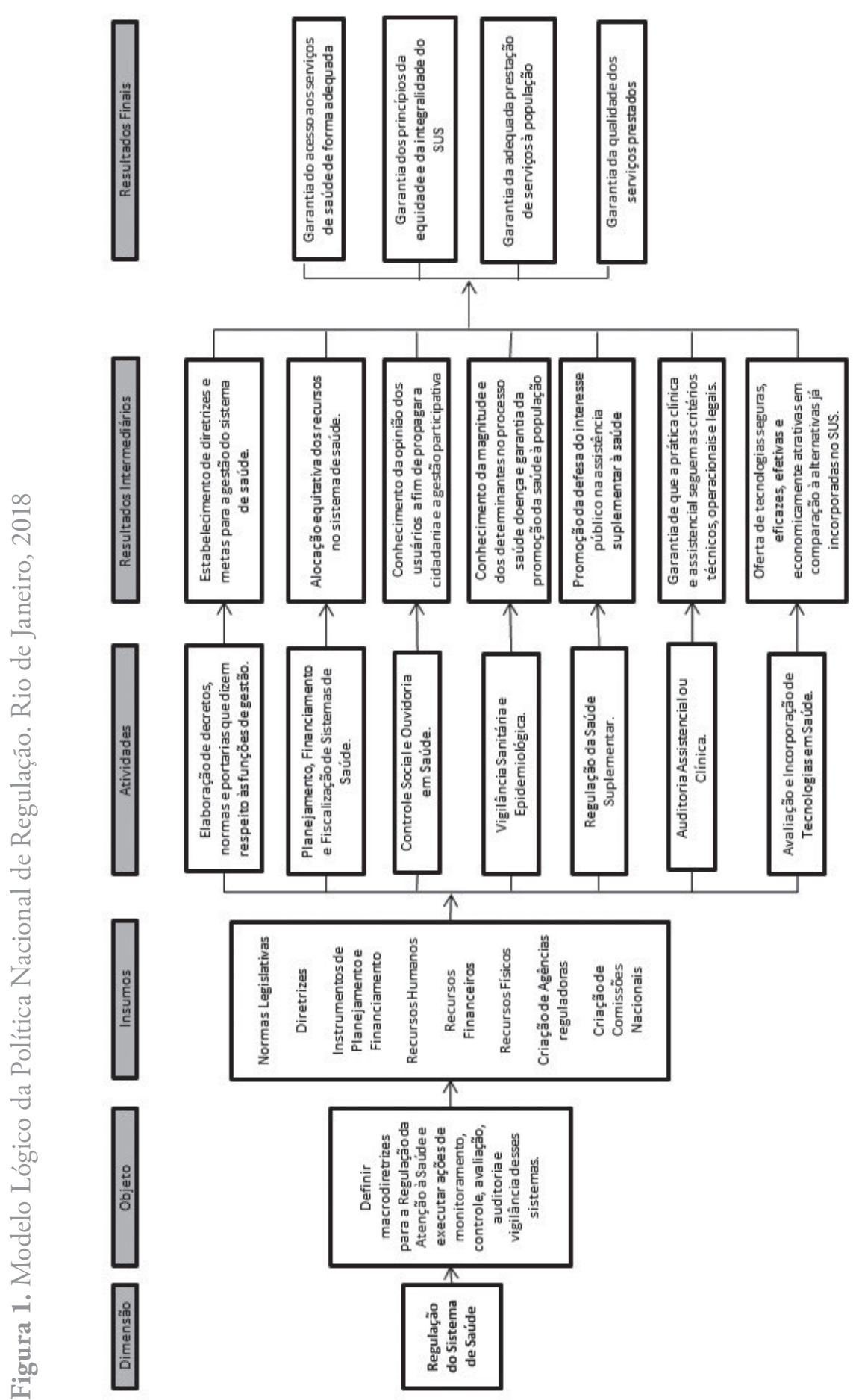




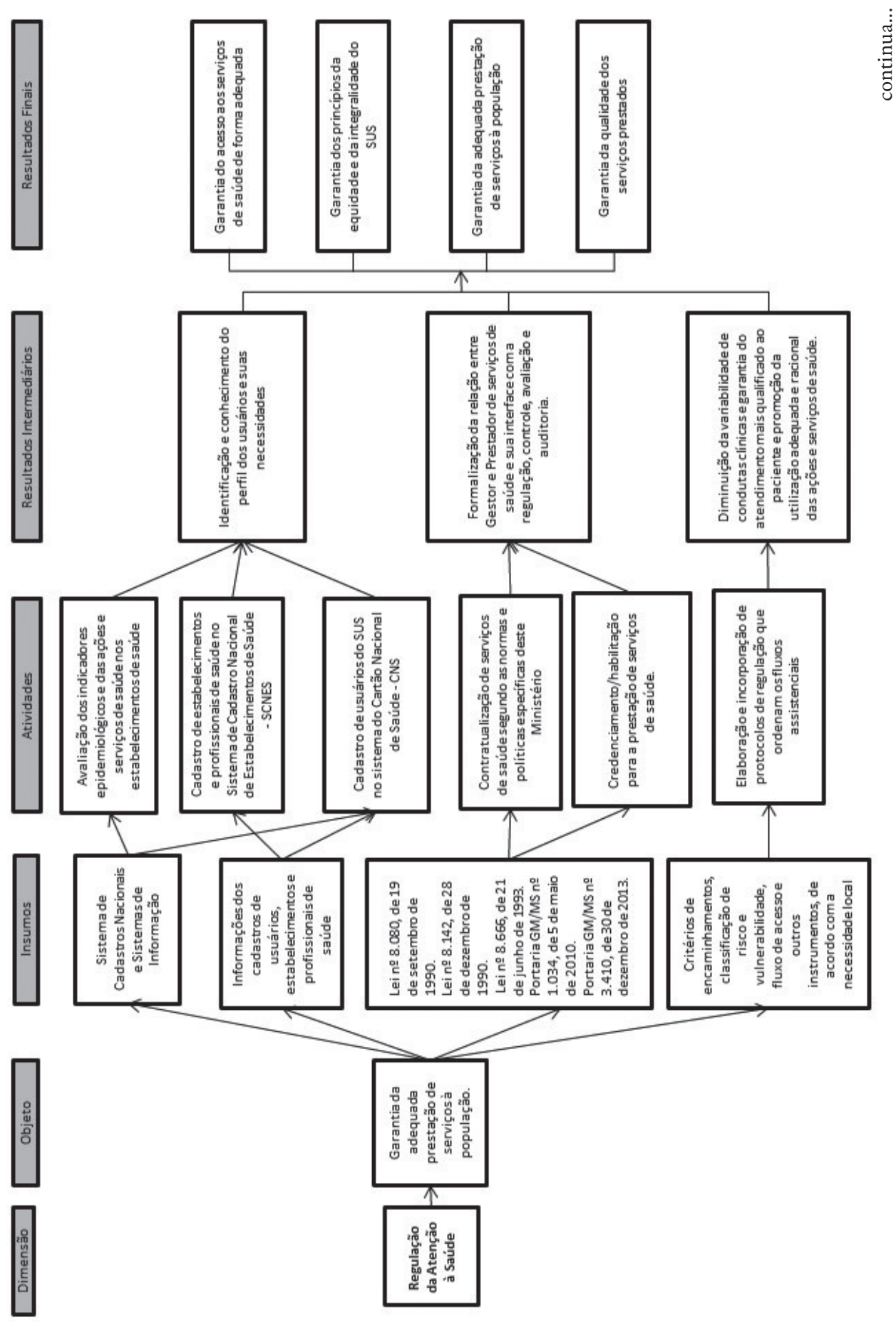




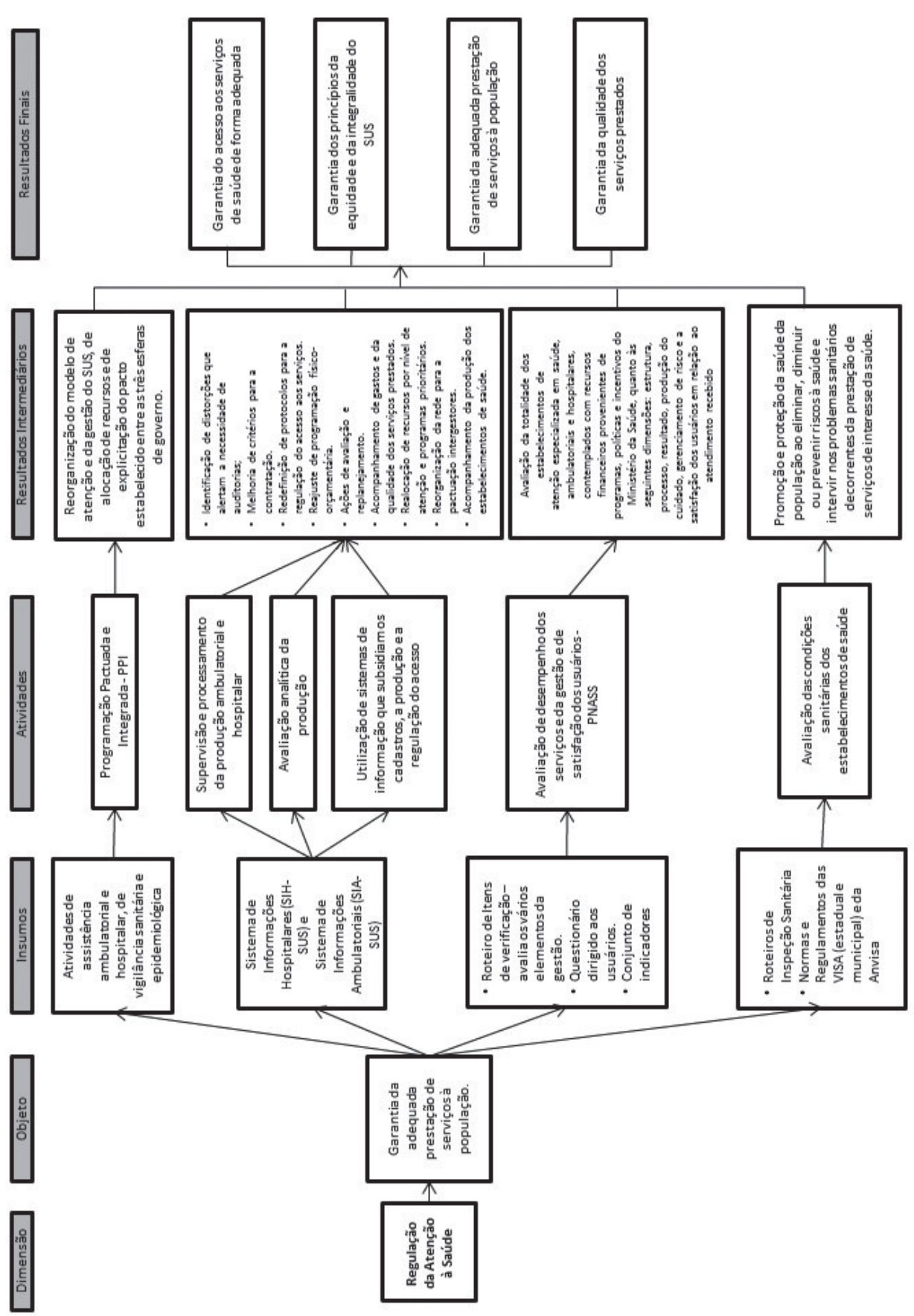



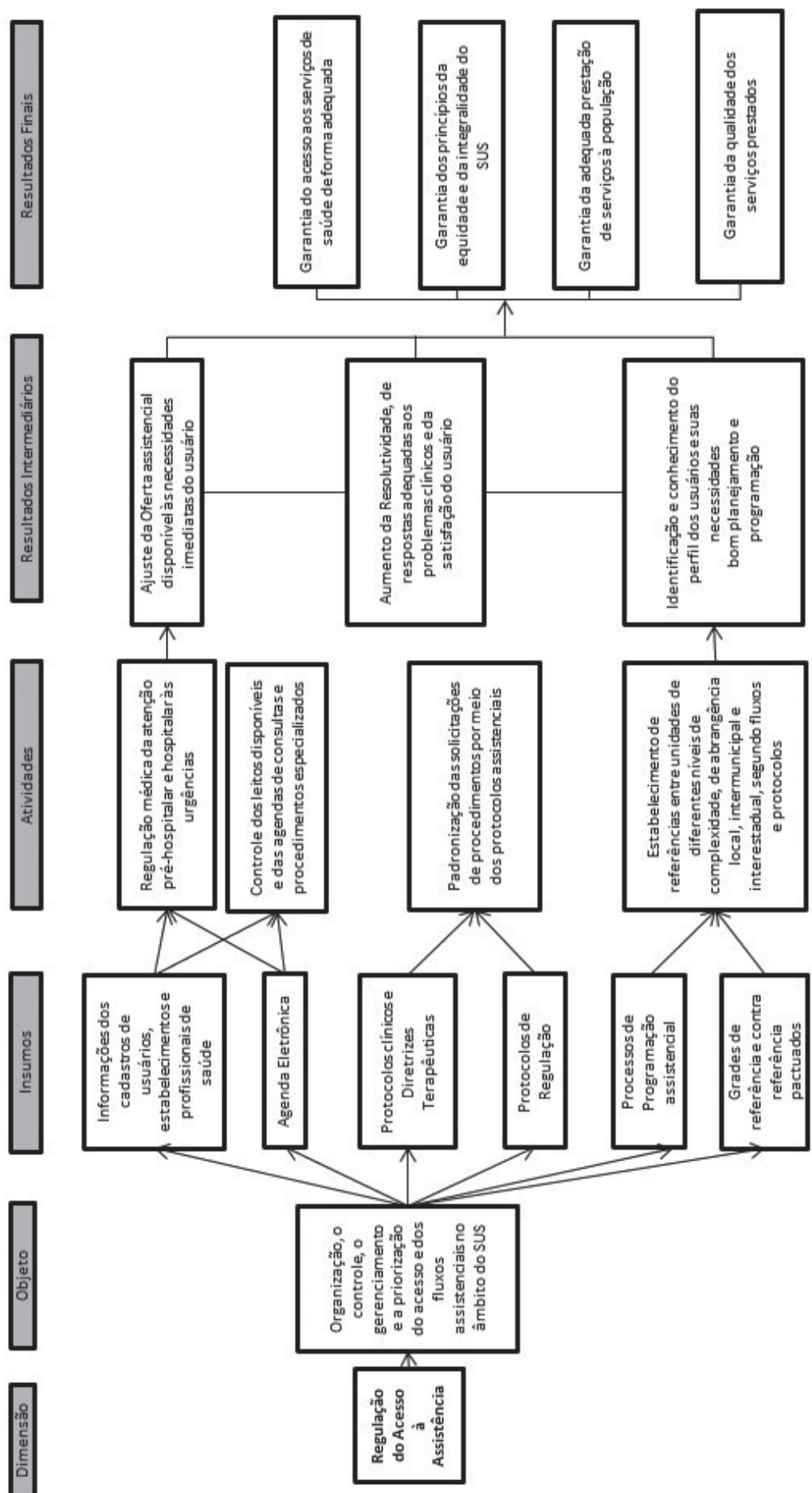

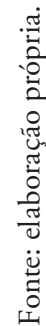


Segundo Silva et al. (2007), o modelo teórico de uma política fundamenta-se, principalmente, na decisão política que antecede sua operacionalização, e é composto: (a) pelo problema que demandou a criação da intervenção; (b) pelo contexto/cenário em que a política se desenvolve; e (c) pelos atores envolvidos no seu planejamento e execução.

A teoria da PNR (figura 2) apresenta a relação entre o contexto externo, o contexto político-institucional, o cenário para implantação e os fatores para sustentabilidade da política. O modelo lógico da política é influenciado pelo contexto políticoinstitucional, sendo que ambos estáo sob influência do contexto externo, que é formado por fatores que levam à necessidade de regulação pelo Estado, como assimetria de informação e falhas de mercado.

\section{Identificação dos usuários e envolvidos na avaliação (elemento 6)}

Segundo os documentos analisados, os interessados na avaliação seriam os gestores municipais, estaduais e federal de saúde, assim como a população usuária do SUS.

\section{Discussão}

Antes da avaliação sistemática de uma política ou um programa, preconiza-se que seja realizado estudo exploratório da situação concreta, ou seja, uma prévia da avaliação propriamente dita, a fim de determinar se a avaliação extensa realmente se faz necessária. Neste sentido, os estudos de avaliabilidade têm ganhado destaque, pois proporcionam exame detalhado da intervenção, contribuindo para estudos avaliativos futuros e ainda para a melhor compreensão e desenvolvimento da intervenção (BEZERRA et al., 2012; SOUZA et al., 2017; DIAS, 2018). O presente estudo de avaliabilidade da PNR contribuiu para o melhor entendimento de sua estrutura e operacionalização, visando alcançar resultados e impactos na saúde.

Em geral, a maioria dos projetos/programas/políticas de saúde apresenta seus objetivos e diretrizes com pouca clareza e falta de consenso entre os atores envolvidos. Assim, os estudos de avaliabilidade mostram-se úteis para o esclarecimento dos objetivos e metas do programa, no desenvolvimento de sua teoria e na identificação de um modelo que permita verificar a plausibilidade em atingir determinados resultados (SOUZA et al., 2017). O presente estudo constatou a mesma dificuldade, ou seja, os objetivos e metas não foram facilmente identificados. 


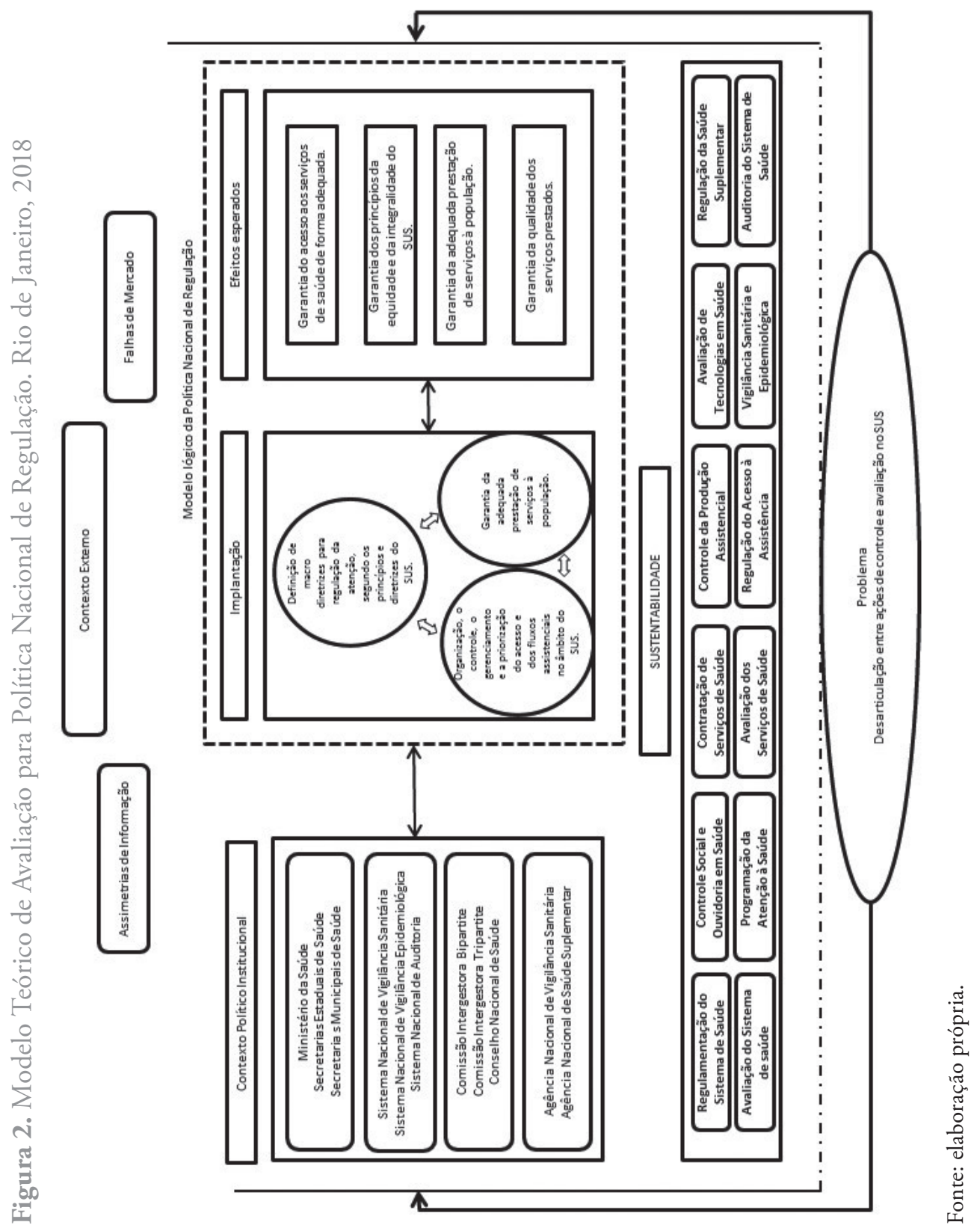


O modelo lógico elaborado constitui-se, portanto, em instrumento bastante útil para compreender e realizar investigaçóes e discussão sobre a PNR. Isso porque se trata de um "conjunto dos postulados sobre o modo pelo qual um programa é relacionado com os benefícios que supostamente produz e a estratégia e as táticas que foram adotadas para alcançar suas metas e objetivos" (MEDEIROS et al., 2010, p. S2010). Importante pontuar que o modelo lógico pode e deve ser revisitado e, a depender da necessidade, pode ser readequado para contemplar novos aspectos ou outros não previstos, que surgem com o desenvolvimento ou aperfeiçoamento da política no decorrer da sua implantação (PADILHA et al., 2015).

O tamanho do modelo lógico apresentado neste estudo demonstra a complexidade da PNR. Desta forma, não foi possível elaborar uma matriz de critérios/indicadores, ainda que seja de grande importância para o processo avaliativo. Cabe pontuar que a utilização da matriz de análise e julgamento oferece apenas uma das possibilidades metodológicas. A escolha do método avaliativo pode ser tão diversificada quanto forem as necessidades e os interesses de informação dos envolvidos na avaliação, devendo-se levar em conta as condições existentes para sua realização (recursos, tempo, oportunidade) (WHOLEY, 1987; PADILHA et al., 2015).

Segundo Wholey (1987), a avaliabilidade permite verificar a viabilidade de processos avaliativos e aumentar as chances de uso de futuras avaliaçóes, na medida em que explora as expectativas e necessidades dos grupos de interesse envolvidos e o grau de organização e implementação da iniciativa. Pode-se, ainda, complementar afirmando que a avaliabilidade permite mapear desacordos entre os grupos de interesse ou destes com os propósitos de um programa ou serviço, sendo especialmente útil na definição de quais aspectos do programa necessitariam ser avaliados (MENDES et al., 2010). Neste sentido, reforça-se a limitação do presente trabalho, por não ter validado os modelos lógico e teórico com envolvidos na avaliação. A falta de validação dos modelos é uma limitação do trabalho, mas não desqualifica o esforço realizado, podendo ser realizado em etapa futura.

No que tange à avaliação de políticas, ainda que haja semelhanças com a avaliação de programas, o nível de análise necessário é superior ao de programas, uma vez que a política é mais ampla e abrange vários programas. Tal fato também dificulta a definição de controle e limites, e ainda torna a escala e o escopo da coleta de dados maiores (CDC, 2018). 
O debate sobre regulação em saúde no Brasil iniciou-se com as Normas Operacionais Básicas - NOB 01/91, 01/92, 01/93 e 01/96 - e a principal tentativa na época foi definir os papéis dos níveis de gestão no processo regulatório (SANTOS; MERHY, 2006). A discussão acerca da regulação da assistência começou a ser aprofundada a partir de 2001, com as Normas Operacionais de Assistência à Saúde (NOAS), em razão da regionalização da assistência, ampliando-se a responsabilidade dos municípios sobre a atenção básica enquanto que os estados e a União focam nos serviços públicos de saúde de média e alta complexidade. Mas é com o Pacto de Gestão (2006) que a regulação ganha força (BRASIL, 2016a; SANTOS; MERHY, 2006). Isso porque busca a autonomia para estados e municípios no que tange aos processos normativos do SUS definiu a responsabilidade sanitária e as atribuiçóes das esferas de governo, fortalecendo, assim, a gestão compartilhada (VILARINS et al., 2012).

Internacionalmente, a primeira onda (década de 1930) de reforma regulatória apresenta-se após o reconhecimento das "falhas de mercado", sendo necessária, assim, a intervenção estatal. Já a segunda onda reformista surge a partir dos anos 1970, em contraposição ao modelo anterior, quando o intervencionismo estatal passou a apresentar sinais de esgotamento no contexto político, econômico e social da época. A abordagem passa a ser focada na liberalização da economia, baseada no redesenho e delimitação do papel e da dimensão do Estado. O debate atual discorre desta posição, pois ao invés de menos regulação ou simples "desregulação", busca-se a "regulação de melhor qualidade", baseada na visão sistêmica do governo e no aperfeiçoamento, difusão e uso mais efetivos de práticas e ferramentas institucionalizadas de transparência, participação e controle social da atividade regulatória, em busca de maior aderência da regulação aos interesses e valores políticos, econômicos e sociais (DIAS, 2012). Nesta visão contemporânea, houve ainda a introdução de novos instrumentos a partir do final da década de 1990, com a regulamentação dos planos de saúde, por meio da Lei no 9.656/98 e, posteriormente, com as criaçóes da Anvisa e da ANS.

Em 2008, a PNR procura aperfeiçoar a utilização dos serviços, em busca da qualidade da açáo, da resposta adequada aos problemas clínicos e da satisfação do usuário, sem que haja a fragmentação do cuidado (VILARINS et al., 2012). Todavia, ela une a antiga mentalidade relacionada à regulação, como a regulação do acesso aos serviços de saúde, a regulação sanitária e a regulação do mercado, com conceitos 
recentes de ouvidoria e controle social, e ainda o comprometimento na prestação de contas e informação para auditorias. Por fim, destacam-se a inclusão da avaliaçáo e a incorporação de tecnologias em saúde.

A avaliação e incorporação de tecnologias em saúde é uma forma de regulação de mercado, no caso, mercado de tecnologias em saúde (medicamentos, vacinas, dispositivos médicos, equipamentos, entre outros). Tal regulação relaciona-se diretamente ao acesso a essas tecnologias, uma vez que a incorporação da tecnologia pelo sistema público de saúde traduz-se em financiamento da mesma. Para além da regulação sanitária, o reconhecimento da avaliação de tecnologias em saúde, como atividade da regulação, é um avanço na compreensão do conceito de regulação e da abrangência desta política.

Diante da complexidade da PNR, cabe ressaltar alguns pontos da regulação em saúde no Brasil. Primeiro, em comparação a outros mercados, o consumo de cuidados de saúde pelo paciente/cliente é diferente, pois há divergência entre o consumidor e o prescritor, e entre o consumidor e o pagador dos serviços. Em outros mercados, é o consumidor que escolhe os produtos ou serviços que quer adquirir. Na saúde, o consumidor (paciente) pode fazer a escolha inicial do médico ou serviço de saúde, mas não tem a mesma liberdade quando a consulta resulta na prescrição de tratamentos, exames, medicamentos ou, simplesmente, novas consultas. Isto significa que é o prescritor que define o que vai ser consumido pelo doente. Em linguagem econômica, diz-se que o médico (ou outro prestador) é um agente do doente, pois faz as escolhas no seu lugar. Uma das partes toma decisóes que não são totalmente controláveis pela outra, mas que influenciam os resultados finais (FIGUEIREDO; HARTZ, 2017; CASTRO, 2002).

Outra questão é o fato de o Estado desempenhar papel de financiador, prestador e regulador, ocasionando conflitos entre os mesmos. A regulaçáo pelo Estado acaba por trabalhar em uma lógica voltada para o controle, com dinâmica própria, restringindo-se às açóes de controle do setor privado conveniado, isolando-se do conjunto das açóes assistenciais e dos serviços próprios. Ainda que haja contratação de entidades privadas como prestadoras, torna-se necessária, nesse contexto, a existência de uma entidade reguladora independente, que desempenhe papel de árbitro e contribua para atenuar os eventuais conflitos de interesses, quer entre as várias funçôes de Estado, quer entre Estado e instituições privadas (FIGUEIREDO; HARTZ, 2017; CASTRO, 2002). 
A regulação em saúde apresenta complexos desafios, principalmente relacionados à eficiência, bem como a normativa relativa à equidade, em um cenário que favorece fracassos de mercado e de Estado. Ademais, problemas relacionados com a informação (confiabilidade principalmente) são particularmente limitativos da eficácia da própria regulação para garantir menores ineficiências e mais equidade (FIGUEIREDO; HARTZ, 2017; CASTRO, 2002).

Pelo mesmo fato de a regulação em saúde não ser como a regulação da economia, que se pauta restritivamente em corrigir falhas de mercado, não pode aplicar modelos de avaliação oriundos desses mercados. Isso porque sua avaliação deve considerar fatores políticos e sociais para além dos técnicos e mercadológicos. Além disso, a regulação em saúde é um processo político, sujeito a pressōes de diferentes grupos, no qual o papel dos usuários deve ser valorizado, de modo a garantir o equilíbrio de poderes, maior transparência e a minimizaçáo do perigo e do envolvimento do regulador pelo ambiente do regulado (FIGUEIREDO; HARTZ, 2017; CASTRO, 2002).

Segundo Felicielo e Vilalba (2016), a regulação em saúde no Brasil vem sendo organizada de modo pulverizado, levando ao surgimento de um conjunto de organismos e instituiçôes que se ocupam de partes, e não do todo da regulação, o que muitas vezes acarreta equívocos e falhas, em razão de uma visão ampla. Além disso, insistem que a regulação em saúde não deve limitar-se às relaçôes econômicas e de mercado, mas levar em conta as políticas setoriais, as formas de financiamento e organizaçôes de serviços de saúde, assim como a oferta e a organização de recursos humanos, o desenvolvimento científico e tecnológico, a inovação e a garantia de acesso aos serviços. Se não for assim, dificilmente a regulação contribuirá para a garantia do direito à saúde.

Para além da regulação em saúde, cabe pontuar dois grandes desafios gerais no Sistema Único de Saúde: a deficiência de financiamento e a gestão, muitas vezes inadequada no sistema de saúde. Dentre esses dois pontos estão problemas crônicos ainda longe de serem resolvidos, como a efetiva regulação de leitos em serviços privados conveniados ou contratados com o sistema público; uma coordenação dos cuidados pela atenção primária nem sempre eficiente; o déficit na oferta de serviços requeridos para satisfação das necessidades de saúde da população; a solicitação sem critérios de serviços especializados, sobretudo no que se refere a situaçôes de urgência e emergência; a fragmentação e os limites da autonomia da regulação (BARROS; AMARAL, 2017). 


\section{Considerações finais}

A avaliação em saúde é fundamental para o processo de tomada de decisão. Ainda que a avaliação esteja entre as açóes da regulação no setor saúde, o ponto norteador deste trabalho foi como avaliar a regulação no SUS.

A Política Nacional de Regulação, conforme apresentada e discutida, possui numerosos componentes e atividades. Assim, considera-se difícil a elaboração de indicadores e de uma matriz de avaliaçáo da política global. Desta forma, sugerese um recorte da política por dimensão ou até mesmo em menor escala, para um possível processo avaliativo que permita identificar de forma adequada os objetivos, as atividades e os resultados esperados pela implantaçáo desta política.

Acredita-se que esta seja uma limitação do presente estudo. Outro ponto limitante que merece destaque refere-se à falta de validaçấo do modelo lógico com os atores envolvidos. Tal processo muito auxiliaria na definição dos objetivos pretendidos, no desenvolvimento de atividades ou grau de implantaçáo dos componentes, e nas estratégias avaliativas orientadas pelos resultados. O estudo de avaliabilidade seguiu as recomendaçóes e as práticas referenciadas pela literatura internacional e teve como sua maior contribuição a melhor compreensão da política em questáo.

Por fim, cabe pontuar que após dez anos da publicação, a PNR não recebeu proposiçóes de avaliação. Sabe-se que a regulação no setor saúde apresenta desafios, principalmente relacionados à eficiência, bem como a normativa relativa à equidade. Para avaliar a regulação em saúde, cabe uma proposta que considere não só as dimensóes de eficiência e equidade, mas a formação e as atividades dos profissionais, a acessibilidade aos serviços e às tecnologias em saúde. Neste sentido, destaca-se a importância do presente estudo, ao apresentar uma proposta preliminar da avaliabilidade da Política Nacional de Regulação e de avaliação da regulação no setor saúde. ${ }^{1}$

\section{Referências}

ALBIERI, F. A. O.; CECILIO, L. C. O. De frente com os médicos: uma estratégia comunicativa de gestão para qualificar a regulaçấo do acesso ambulatorial. Saúde Debate. Rio de Janeiro, v. 39, p. 184-195, 2015.

ALBUQUERQUE, N. Avaliação do acesso a leitos de UTI através da Central de Regulação Hospitalar do SUS em PE no ano de 2009. 48f. Monografia (Especialização) - Centro de Pesquisas Aggeu Magalhães, Fundação Oswaldo Cruz, Recife, 2010. 
ALVES, F. N. R.; PECI, A. Regulatory Impact Analysis: a new tool for better regulation at ANVISA. Revista de Saúde Pública. São Paulo, v. 45, n. 4, p. 802-805, 2011.

BARBOSA, D. V.S.; BARBOSA, N.B.; NAJBERG, E. Regulação em Saúde: desafios à governança do SUS. Cadernos de Saúde Coletiva. Rio de Janeiro, v. 24, n. 1, p. 49-54, 2016.

BARROS, F. P. C.; AMARAL, T. C. L. Os desafios da regulação em saúde no Brasil. Anais do Instituto de Higiene e Medicina Tropical. Lisboa, v. 16, supl. 3, p. S39-S45, 2017.

BARROSO, J. O estado, a educação e a regulação das políticas públicas. Educação e Sociedade. Campinas, v. 26, n. 92, p. 725-751, 2005.

BEZERRA, L. C. A. et al. Identificação e caracterização dos elementos constituintes de uma intervenção: pré-avaliação da Política Participa SUS. Ciência \& Saúde Coletiva. Rio de Janeiro, v. 17, n. 4 , p. $883-900,2012$.

BRASIL. Ministério da Saúde. Departamento Nacional de Auditoria do SUS Coordenação-Geral de Desenvolvimento, Normatização e Cooperação Técnica. Orientaçóes Básicas para utilização de Sistemas informatizados em auditoria no SUS. Brasília, 2007. Disponível em: http://sna.saude. gov.br/download/Orientacoes\%20basicas\%20para\%20utilizacao\%20de $\% 20$ Sistemas\%20 Informatizados\%20em\%20Auditoria\%20no\%20SUS.pdf. Acesso em: 16 dez. 2018.

. Ministério da Saúde. Portaria no 1.559, de $1^{\text {o }}$ de agosto de 2008. Institui a Política Nacional de Regulação do Sistema Único de Saúde - SUS. Diário Oficial da União. Brasília, Seção 1, 4 ago. 2008.

Ministério da Saúde. Secretaria de Atenção à Saúde Departamento de Regulação, Avaliação e Controle de Sistemas. Diretrizes para a Implantação de Complexos Reguladores. Brasília, 2006. Disponível em: http://bvsms.saude.gov.br/bvs/publicacoes/ DiretrizesImplantComplexosReg2811.pdf. Acesso em: 20 dez. 2018.

- Ministério da Saúde. Secretaria de Atenção à Saúde Departamento de Regulação, Avaliação e Controle de Sistemas. Manual Técnico Operacional do Sistema de Informação Hospitalar do SUS. Brasília, 2012 Disponível em: http://bvsms.saude.gov.br/bvs/publicacoes/ manual_tecnico_sistema_informacao_hospitalar_sus.pdf. Acesso em: 20 dez. 2018.

- Ministério da Saúde. Secretaria de Atenção à Saúde Departamento de Regulação, Avaliação e Controle de Sistemas. Manual de Orientaçôes para Contratação de Serviços de Saúde. Brasília, 2016b. Disponível em: http://portalarquivos.saude.gov.br/images/ pdf/2016/novembro/06/MANUAL-DE-ORIENTACOES-PARA-CONTRATACAO-DESERVICOS-DE-SAUDE.pdf. Acesso em: 20 dez. 2018.

- Ministério da Saúde. Secretaria de Atenção à Saúde. Departamento de Regulação, Avaliação e Controle de Sistemas. Curso Básico de Regulação do Sistema Único de Saúde. Brasília, 2016a. Disponível em: http://bvsms.saude.gov.br/bvs/publicacoes/curso_regulacao_SUS_1ed_ eletronica.pdf. Acesso em: 20 dez. 2018. 
. Ministério da Saúde. Secretaria de Gestão Estratégica e Participativa. Departamento de Ouvidoria-Geral do SUS. Manual das Ouvidorias do SUS. Brasília, 2014. Disponível em: http:// bvsms.saude.gov.br/bvs/publicacoes/manual_ouvidoria_sus.pdf. Acesso em: 20 dez. 2018.

CASTRO, J. D. Regulação em saúde: análise de conceitos fundamentais. Sociologias, Porto Alegre, v. 4, n. 7, p. 122-36, 2002.

CENTER FOR DISEASES CONTROL AND PREVENTION (CDC). Policy Evaluation. Step by Step: Evaluating Violence and Injury Prevention Policies. Brief 1: Overview of Policy Evaluation. Disponível em: https://www.cdc.gov/injury/pdfs/policy/Brief\%201-a.pdf. Acesso em: 20 dez. 2018. CONSELHO NACIONAL DE SECRETÁRIOS DE SAÚDE. Regulação em Saúde. In: . Coleção Para Entender a Gestão do SUS. Brasília: CONASS, 2011. Disponível em: http://www.conass.org.br/bibliotecav3/pdfs/colecao2011/livro_10.pdf. Acesso em: 20 dez. 2018. DIAS, M. S. A. et al. Política Nacional de Promoção da Saúde: um estudo de avaliabilidade em uma regiáo de saúde no Brasil. Ciência \& Saúde Coletiva. Rio de Janeiro, v. 23, n. 1, p. 103-114, 2018.

DIAS, R. L. Regulação médica de urgências na região do Médio Paraíba: uma proposta para avaliação da implantação do serviço. 99f. Dissertação (Mestrado) - Instituto de Medicina Social, Universidade do Estado do Rio de Janeiro, Rio de Janeiro, 2012.

FELICIEllO, D.; VIlAlBA, J. P (Org). Regulação em saúde no SUS. Campinas, 2016. Disponível em: <https: //www.nepp.unicamp.br/upload/documents/publicacoes/015fddb848e 50e0e8e990045afd87e04.pdf>. Acesso em: 20 dez. 2018.

FERREIRA, J. B. B. et al. The regulatory complex for healthcare from the perspective of its operational players. Interface - Comunicação, Saúde e Educação. Botucatu, v. 14, n. 33, p. 345-58, 2010.

FIGUEIREDO, T. A.; HARTZ, Z. Avaliação de desempenho da regulação em saúde. Anais do Instituto de Higiene e Medicina Tropical. Lisboa, v. 16, supl 3, p. S19-S28, 2017.

GIANNOTTI, E. M. A organização de processos regulatórios na gestão municipal de saúde e suas implicaçóes no acesso aos serviços: um estudo de caso do município de Guarulhos. 132 f. Dissertação (Mestrado) - Faculdade de Saúde Pública, Universidade de São Paulo, São Paulo, 2013.

GUETTERMAN, T.; HARE, J. Principal Components Analysis of Evaluability Assessments: An Empirical Study. The EES Newsletter Evaluation Connections, 2014. Disponível em: https://www.europeanevaluation.org/sites/default/files/ees_newsletter/ees-newsletter-2014-10november-final.pdf. Acesso em: $20 \mathrm{dez} .2018$.

HARTZ, Z. M. A.; VIEIRA-DA-SILVA, L. M. Avaliação em saúde: dos modelos teóricos à prática na avaliação de programas e sistemas de saúde. Rio de Janeiro: Editora Fiocruz; 2005. 
LEVITON, L. C. et al. Evaluability assessment to improve public health policies, programs, and practices. Annual Review of Public Health. Palo Alto, v. 31, p. 213-33, 2010.

MACHADO, C. V. O modelo de intervenção do Ministério da Saúde brasileiro nos anos 90. Cadernos de Saúde Pública, Rio de Janeiro, v. 23, n. 9, p. 2113-2126, 2007.

MEDEIROS, P. F. P. et al. Um estudo sobre a avaliabilidade do Programa + Vida: política de redução de danos em álcool, fumo e outras drogas do município de Recife, Brasil. Revista Brasileira de Saúde Materno-Infantil. Recife, v. 10, n. 1, p. S209-S217, 2010.

MENDES, M. F. M. et al. Avaliabilidade ou Pré-avaliação de um Programa. In: SAMICO I. et al. (Orgs.). Avaliação em Saúde: bases conceituais e operacionais. Rio de Janeiro: MedBook; 2010. p.57-64.

NASCIMENTO, L. V. et al. Estudo de avaliabilidade da Política Nacional de Atenção Integral à Saúde do Homem no Município de Sobral, Ceará. Revista Baiana Saúde Pública. Salvador, v. 38, n. 1, p. 95-114, 2014.

OCKÉ-REIS, C. O. Sustentabilidade do SUS e renúncia de arrecadação fiscal em saúde. Ciência \& Saúde Coletiva. Rio de Janeiro, v. 23, n. 6, p. 2035-42, 2018.

OLIVEIRA, R. R.; ELIAS, P. E. M. Conceitos de regulação em saúde no Brasil. Revista de Saúde Pública. São Paulo, v. 46, n. 3, p. 571-6, 2012.

PADilha, M. A., OliveirA, C. M., FIGUEIRÓ, A. C. Estudo de avaliabilidade do Programa Academia Carioca da Saúde: desafios para a promoção da saúde. Saúde em Debate. Rio de Janeiro, v. 39, n. 105, p. 375-386, 2015.

PEITER, C. C.; LANZONI, G. M. M.; OLIVEIRA, W. F. Regulação em saúde e promoção da equidade: o Sistema Nacional de Regulação e o acesso à assistência em um município de grande porte. Saúde em Debate. Rio de Janeiro, v. 40, n. 111, p. 63-73, 2016.

SALGADO, L. H. Agências regulatórias na experiência brasileira: um panorama do atual desenho institucional. Brasília, 2003. Disponível em: http:/www.ipea.gov.br/portal/images/ stories/PDFs/TDs/td_0941.pdf. Acesso em: Acesso em: 20 dez. 2018.

SANTOS, F. P.; MERHY, E. E. A regulação pública da saúde no Estado brasileiro: uma revisão. Interface: Comunicação, Saúde e Educação. Botucatu, v. 10, n. 19, p. 25-41, 2006.

SCHILling, C. M.; REIS, A. T.; MORAES, J. C. (Orgs.). A politica regulação do Brasil. Brasília, 2006. Disponível em: from: http://portalarquivos2.saude.gov.br/images/pdf/2015/ marco/09/DesenvolvimentodeSistemaseServicosdeSaude.pdf>. Access: April 10, 2018.

SILVA, G. H. T. Performance regulatória: uma análise do Programa de Melhoria do Processo de Regulamentação da Anvisa no contexto da atual Agenda de Reforma Regulatória no Brasil. 294f. Dissertação (Mestrado) - Escola Nacional de Saúde Pública, Fundação Oswaldo Cruz, Rio de Janeiro, 2013. 
SILVA, L. M. V. Avaliação de políticas e programas de saúde. Rio de Janeiro: Fiocruz; 2014.

SILVA, R. C. A avaliabilidade do Programa SESI de prevenção de quedas na indústria de construção civil na Bahia. Dissertação (Mestrado) - Universidade Federal da Bahia, Salvador, 2007.

SIMÓES, J.; CARNEIRO, C. Investigação avaliativa na regulação da saúde. Anais do Instituto de Higiene e Medicina Tropical. Lisboa, v. 16, supl. 3, p. S81-S86, 2017.

SOUZA, E. C.; GUIMARÃES, J. M. X.; SILVA, M. R. F. Estudos de avaliabilidade de políticas e programas de saúde no Brasil: revisão integrativa. SANARE - Revista de Políticas Públicas. Sobral, v. 16, v. 2, p. 85-92, 2017.

THURSTON, W. E.; RAMALIU, A. Evaluability assessment of survivors of torture program: lessons learned. Canadian Journal of Program Evaluation. Renfrew, v. 20, n. 2, p. 1-25, 2005.

VILARINS, G. C. M.; SHIMIZUI, H. E; GUTIERREZ, M. M. U. A regulação em saúde: aspectos conceituais e operacionais. Saúde em Debate. Rio de Janeiro, v. 36, n. 95, p. 640-647, 2012. WHOLEY, J. S. Evaluability assessment: Developing program theory. New Directions for Program Evaluation. Medford, v. 33, p. 77-92, 1987.

\section{Nota}

${ }^{1}$ T. A. Figueiredo participou da concepção da ideia central do projeto de pesquisa, coleta e análise de dados e da redação do artigo. A. Angulo-Tuesta contribuiu com a análise dos dados e participou da redação e revisão da versão final do artigo. Z. Hartz participou da concepção da ideia central do projeto de pesquisa e da redação e revisão da versão final do artigo. 


\section{Abstract}

\section{Evaluability of the Brazilian Regulatory}

Policy within the SUS: a preliminary proposal

Evaluability studies allow determining the purposes of the policy and the evaluation focus, assisting a thorough understanding and prior appreciation of the possibilities of policy evaluation. Thus, this article analyzes the evaluability of the Brazilian Regulatory Policy, from January to May of 2018, with an exploratory and qualitative approach, in order to define how this policy can be evaluated and the elaboration of the logical and theoretical models of evaluation, according to the sevenelement system by Thurston and Ramaliu. The results contributed to a better understanding of the structure and operationalization of the policy to reach their results and impacts on health. However, the Policy has many components and activities, so it is difficult to elaborate indicators and a global policy assessment matrix. It is suggested a cut of the policy for an evaluation process. The Brazilian Regulatory Policy was published 10 years ago but any proposal to evaluate it has been submitted. It is known that regulation in the health sector brings numerous challenges, and it requires a proposal of evaluation that goes beyond the dimensions of efficiency and equity, including the training and activities of health professionals, accessibility to health services and health technology assessment.

> Keywords: health evaluation; health policies; healthcare coordination and monitoring; health system. 